\title{
Concepción de los criterios de creatividad de los contadores públicos de la ciudad de Villavicencio en su quehacer
}

\section{Conception of the creativity criteria of the public accountants of the city of Villavicencio in their work}

Como citar este artículo / To reference this articcle: Hernández-González José W., Delgadillo-Chavez Natalia, \& Gaitán-Ortiz Carolina (2021). Concepción de los criterios de creatividad de los contadores públicos de la ciudad de Villavicencio en su quehacer EL CONUCO: (investigación, economía y sociedad), 3(2), pp. 1-13. DOI: https://doi.org/10.22579/2619-614X.778

José William Hernández González

Natalia Delgadillo Chavez ${ }^{2}$

Carolina Gaitán Ortiz ${ }^{3}$

\section{Artículo de investigación}

Recepción: 14-12-2020

Aceptación: 13-04-2021

El Conuco es una revista de acceso abierto revisada por pares. (C) $2018 \mathrm{El}$ autor (es). Este es un artículo de acceso abierto distribuido bajo los términos de la Licencia Internacional Creative Commons Attribution 4.0 (CC-BY 4.0), que permite el uso, distribución y reproducción sin restricciones en cualquier medio, siempre que se acredite el autor y la fuente originales.

Consulte http://creativecommons.org/licenses/by/4.0/. ऽOPEN ACCESS

(c) (1) $(9)$

$1 \mathrm{PhD}$. Investigación Cualitativa. Docente Ocasional tiempo completo del programa de Contaduría Pública, Facultad Ciencias Económicas, Universidad de los Llanos, correo institucional: jwhernandez@unillanos.edu.co, ORCID: ID https://orcid.org/0000-0003-1173-5021

2 Estudiante de VI semestre, programa de Contaduría Pública, Facultad Ciencias Económicas, Universidad de los Llanos, correo institucional: natalia.delgadillo@unillanos.edu.co, ORCID: ID https://orcid.org/0000-0002-1491-8731

3 Estudiante de VI semestre, programa de Contaduría Pública, Facultad Ciencias Económicas, Universidad de los Llanos, correo institucional: carolina.gaitan.ortiz@unillanos.edu.co, ORCID: ID https://orcid.org/0000-0002-1736-4420

\section{Resumen}

El propósito de este artículo es presentar una aproximación teórica sobre la contabilidad creativa como objeto de estudio; donde la etimología del término creatividad ha sido desfigurada en la disciplina contable, homologando este concepto con el de fraude, siendo interpretada de forma errónea, asociándolo con aquellos hechos fraudulentos que realiza el profesional al manipular sus saberes.

De conformidad con lo anterior, esta investigación se centra en describir la aplicación de los criterios de creatividad por parte de los profesionales de las ciencias contables de la ciudad de Villavicencio en su ejercicio, en los últimos cinco años. Para el desarrollo del estudio se emplearon instrumentos como entrevistas y análisis de documentos obtenidos de bases de datos y repositorios.

Palabras clave. Contabilidad, Creatividad, Fraude, Conocimiento, Ética, Innovación.

Códigos JEL: D83, M41, O35

\section{Abstract}

The purpose of this article is to present a theoretical approach on creative accounting as an object of study; where the etymology of the term creativity has been distorted in 
the accounting discipline, homolocating this concept with that of fraud, being erroneously interpreted, associating it with those fraudulent acts carried out by the professional when manipulating their knowledge.

In accordance with the above, this research focuses on describing the application of creativity criteria by professionals of the accounting sciences of the city of Villavicencio in their exercise, in the last five years. Instruments such as interviews and analysis of documents obtained from databases and repositories were used for the development of the study.

Keywords. Accounting, Creativity, Fraud, Knowledge, Ethics, Innovation.

\section{Introducción}

La contabilidad creativa, que para los asuntos de este artículo se utilizara la abreviación CC, es la habilidad que permite mejorar la práctica contable, a partir de la búsqueda de consolidación de un conocimiento contable orientado hacia el bien común, en donde ésta le concede al experto la capacidad de experimentar y generar ideas que faciliten su ejercicio profesional; aun así el especialista un tiempo atrás ha percibido cierto grado de confusión al momento de definir la contabilidad creativa, pues este concepto se ha distorsionado y su interpretación ha sido manipulada de forma errónea, en donde quienes no tienen conocimientos del ámbito contable la asocian inmediatamente con el fraude, esto se debe a que el concepto de la palabra creatividad es polisémica y genera desatino a la hora definirla en el área contable, por esta razón, el profesional se ha visto sumergido en un entorno de duda e intranquilidad desde ambiente en el cual se desempeña.

Sin juicios de valor o sin criterios facticos, algunos profesionales de la ciencia contable se han dejado manipular, actuando de manera ilícita, violando las leyes y maniobrando el sistema de control, que ponen en riesgo la ética del contador público, además de dañar y poner en tela de juicio los principios de dignificar la profesión y muchas veces el colegage, como también el descredito al buen nombre de la profesión.

En relación con lo antes mencionado, cabe resaltar que el especialista del área contable debe ser una persona íntegra, que está en constante actualización para así lograr permanecer dentro del mundo de la ciencia contable y financiera, pero siempre en el contexto laboral y empresarial donde ejerce la profesión, todo en busca de estar dispuesto a enfrentar cualquier situación que se presente de tipo económico o social, en búsqueda de un interés general, más no individual; aprovechando así el conocimiento adquirido para aportar de manera digna y aplicando la buena fe en la profesión.

El ejercicio de la profesión como Contadores Públicos exige mucha responsabilidad al dar asesorías y desempeñar labores referentes a la Contaduría por lo que se hace muy necesario e importante actualizar los conocimientos en este aspecto para un buen desempeño de la labor. (Gerencie.com, 09 de febrero de 2012, párr. 7)

Con relación con lo antes expuesto, como se menciona en Gerencie.com, es sumamente necesario para el profesional ser un individuo constantemente actualizado en sus conocimientos, donde le permitan evolucionar y desarrollar habilidades, para mantenerse dentro del mercado competitivo en el que se encuentra la profesión, logrando un buen desempeño en el ejercicio profesional.

Por otro lado, la investigación realiza una disertación sobre la definición de contabilidad creativa desde un marco teórico, mostrando la realidad de 
la concepción de creatividad. Este estudio pretende describir en un documento la relación de los criterios de creatividad de los contadores en ejercicio y la literatura que contribuye como referencia.

El proceso metodológico está fundamentado en un carácter cualitativo, con un enfoque descriptivo; tomando como unidad de análisis la profesión desde el punto de vista de las ciencias contables, en su ejercicio y en formación, de la ciudad de Villavicencio, Meta.

\section{Descripción y justificación del problema}

La concepción de contabilidad creativa desde sus inicios se le dio una orientación hacia el fraude, donde resulta difícil esclarecer en qué momento se enrarece este concepto, llevando a un mal uso la definición de la palabra creatividad, siendo deformado este término, en el que anteriormente se concebía como una habilidad de crear, innovar y generar ideas que permitían facilitar y mejorar lo existente; y que actualmente es interpretada en el ejercicio contable de forma desacertada. El problema de esta interpretación se ha dado desde hace un tiempo atrás, autores como Griffiths (1988, como se citó en Santos, 2016), afirma que:

En el Reino Unido, todas las empresas del país han estado escondiendo sus beneficios; señala que las cuentas anuales se basan en libros que han sido manipulados; el autor califica dichas manipulaciones como que han sido "cocinados" o "completamente asados". Indica que las partidas que se muestran dos veces al año al público inversor han sido cambiadas para proteger al culpable y que de hecho este fraude es completamente legítimo, o sea, que es la contabilidad creativa. (p. 48)

De acuerdo a lo anterior, emana del interés de comprender cómo los contadores aplican los cri- terios de Control de Calidad en su entorno laboral, dando a conocer dos posturas que surgen a raíz de esta práctica, la generación de conocimiento y fraude; en este sentido, para abordar el objeto de estudio, se han desarrollado investigaciones, Ruiz (2008), quien realizó un estudio de la creatividad y las normas internacionales de cara a la contabilidad creativa; de la misma manera Bravo (2011), argumenta en su estudio que la contabilidad creativa en "su propio nombre lleva implícito la condición de 'crear', cuando la contabilidad tiene como principal función el reflejo de la realidad" (p. 35). Las cuales permiten visualizar la trascendencia que ha tenido a lo largo de los años la relación del concepto de creatividad en la contabilidad.

Por otra parte, es evidente que el problema de interpretación del término creatividad, afecta principalmente al mismo profesional de las ciencias contables, y a los que se están formando, al homologarse el concepto de creatividad con el de fraude, creando también en el profesional un entorno de duda e intranquilidad desde el medio en el que se desempeña; y cuando esto sucede, según Donal (como se citó en Arbeláez, et al. 2013), "la oportunidad de fraude surge cuando los controles son débiles y/o cuando los individuos se encuentran en una posición de confianza, cuando existe presión de tipo financiero, para cumplir con los objetivos...” (p. 219).

Con relación a lo que anterior, se puede decir que los estudios realizados muestran información acerca de la aplicación de los criterios de creatividad, de los cuales se percibe que la creatividad no sólo es utilizada para la generación de nuevos conocimientos y desarrollo de investigaciones que permiten la actualización del profesional contable, para el buen ejercicio de la profesión, sino que también es una de las principales causas de generación de fraude en consecuencia de 
los vacíos que se presentan en las normas tributarias y las NIC y las NIIF, las cuales regulan el ejercicio de la profesión; enrareciendo el concepto de creatividad, para utilizarlo a su beneficio y distorsionar la información favoreciéndose de la misma, infringiendo la ética profesional para dar muchas veces información errónea o alterada, de esta forma no se cumplirían en especial los principios del Contador Público, de dar confianza y fe pública ante los usuarios. En este sentido, el problema se limita en el departamento del Meta, especialmente en la ciudad de Villavicencio, donde se pretende conocer e interpretar la aplicación del concepto de creatividad en el quehacer contable.

Esta pesquisa ha surgido como una línea de investigación relevante en contabilidad y la ética profesional donde se pretende analizar la percepción del profesional acerca de la utilización e implementación de creatividad en su actuar, visibilizando entonces, dos posturas sobre este objeto de estudio, una de ellas utilizar la creatividad como si fuese un sinónimo de fraude y la otra buscar que la creatividad sea una herramienta que conduzca al profesional de las ciencias contables hacia la generación de conocimiento.

Para dar respuesta al objeto de estudio se hace imprescindible estudiar la arqueología (epistemología) del concepto de creatividad para mediar entre las posturas que se presentan desde las perspectivas de distintos autores. Por otra parte, esta investigación pretende construir un espacio para la comprensión conceptual y la discusión de un fenómeno presente en la profesión.

Cabe mencionar que la manipulación contable o contabilidad creativa, mirándola desde la perspectiva del fraude es un tema de la actualidad, que es una práctica muy extendida pero poco combatida, afecta de manera directa la profesión y al mismo tiempo la disciplina.
Es evidente que algunos contadores públicos se han visto involucrados en la mala práctica de la contabilidad, que atenta de forma directa la integridad profesional, llegando a tal punto que es generalizado este actuar del contador público por parte de la sociedad, como aquel encargado de generar hecho de dolo, maquillando información a conveniencia personal o de terceros; y no como un profesional competente capaz de producir conocimiento, que contribuya a un mejor desempeño de la profesión.

De acuerdo con el apartado anterior se formula la siguiente pregunta de investigación: ¿Cómo aplican los contadores públicos en ejercicio, los criterios de creatividad en su quehacer, en la ciudad de Villavicencio en los últimos 5 años?

\section{Un acercamiento a las Teorías y los Conceptos}

Como soporte del objeto de estudio se tienen en cuenta teorías como contabilidad, conocimiento y creatividad.

Con relación a la contabilidad como disciplina, Ospina (2004), plantea que:

El término de disciplina designa el fundamento investigativo de cada una de las áreas del saber mencionadas y son como la puerta de entrada a esas áreas, pues son las disciplinas las que producen y alimentan el conocimiento. Podríamos afirmar que las disciplinas surgen de la manera como enfocamos aspectos determinados de la realidad que queremos conocer. (p. 2)

De acuerdo a lo anterior, es posible decir que la contabilidad siempre ha ido encaminada a la generación de conocimiento y a la revelación de una realidad veraz, es así como lo señala Ruiz (1995, como se citó en Villarreal, 2009), quien atestigua que "la teoría contable es un conjunto 
coherente de principios hipotéticos, conceptuales y pragmáticos que forman el marco conceptual de referencia, mediante el cual la práctica contable puede ser evaluada y dirigida al desarrollo de nuevas prácticas y procedimientos" (p. 171). Acorde a lo antes mencionado, se puede añadir que la contabilidad también busca la explicación y comprensión de procesos, sucesos y operaciones, es así como lo expresa Gómez (2008), quien señala que la disciplina contable hace referencia al estudio de los principios, fundamentos y métodos del conocimiento que permitan explicar y comprender los procedimientos, técnicas y prácticas desarrolladas en el área contable.

Es importante mencionar, que la contabilidad como disciplina estudia hechos ocurridos en el pasado y los que están ocurriendo en el presente, para proyectar una serie de procedimientos que permitan optimizar el mejoramiento y crecimiento a futuro, es así como lo expresa Cañibano (1997 como se citó en Abbate et al., 2000), enuncia que:

La contabilidad es una ciencia de naturaleza económica y jurídica, cuyo objeto es el conocimiento del pasado, presente y futuro de la realidad económica, en termino cuantitativos y su aplicación normativa, a todos sus niveles organizativos, mediante métodos específicos, apoyados en bases suficientemente contrastadas, a fin de elaborar una información que cubra las necesidades financieras, jurídicas y las de planificación y control internas. (p. 117)

También es posible identificar que al hablar de la teoría contable, se está haciendo referencia a un conjunto de normas, las cuales rigen y orientan al profesional, para que al final se pueda obtener como resultado una información pertinente y fidedigna, es así como lo señalan autores como Gil (2007, como se citó en Mejía et al, 2010), quien manifiesta que "El dominio del derecho contable está dado por un sistema de normas que prescriben acciones, procedimientos y actitudes que culminan en una representación de la realidad económica, financiera y patrimonial emergente de las transacciones en los entes-empresas" (p. 66).

En otro orden de ideas, el conocimiento como base no solo de la ciencia si no de la práctica misma de la contabilidad, se da porque este es objetivo, analiza la realidad de los hechos, además comprende y adquiere información a partir de experiencias; el cual genera el desarrollo intelectual de un individuo; autores como Flores, et al. (2013), consideran que:

El fenómeno del conocimiento radica en la capacidad de la inteligencia humana de examinar la realidad, objetiva e inmanente, y conceptualizarla captando sus características esenciales, generales y particulares, estableciendo una suerte de autorreflexión acerca de lo que logramos aprehender de la cosa, hecho, fenómeno, circunstancia, símbolo o idea conocida. (párr. 23)

De acuerdo con lo expuesto anteriormente, Ramírez (2009), se refiere al conocimiento como un proceso mediante el cual le permite al individuo aprehender, evolucionar y desarrollar habilidades, de tal forma que este pueda adaptarse al entorno cambiante en el que se encuentra la sociedad. Además, es posible afirmar, que el proceso de conocimiento debe ir enlazado con la lógica, la cual estudia los métodos y principios que permiten identificar al razonamiento lo que es correcto frente a lo que no lo es, y de esta forma poder resolver problemas, sacar conclusiones y aprehender de las situaciones que se presentan a diario en la sociedad; según García (2006):

Conocer es organizar los datos de la realidad, darles un sentido, lo cual significa construir una lógica, no la lógica de los textos, sino una lógica 
de la acción, porque organizar es estructurar, es decir, hacer inferencias, establecer relaciones. Estructura es lógica. (p. 119)

El individuo al encontrarse en un entorno cambiante y en constante desarrollo, siente la necesidad de estar actualizado, lo que genera la obtención de nuevos conocimientos que le permitan la adaptación a este; en contabilidad, el conocimiento y la actualización se hace imprescindible para el óptimo desarrollo de la profesión; Pérez y Pinto (2016), mencionan que el conocimiento es una habilidad que obliga al profesional a estar actualizándose constantemente sobre su área de profesión, para así poder competir y aportar a la disciplina de manera honesta.

En vista de que el objeto de estudio de la investigación es la contabilidad creativa, se requiere realizar una aproximación epistemológica del concepto de creatividad, vista a partir de algunos teóricos como Gervilla (1992, como se citó en Esquivas, 2004), quien plantea que "Creatividad es la capacidad para generar algo nuevo, ya sea un producto, una técnica, un modo de enfocar la realidad" (p. 6). De este modo, se puede manifestar que el ser humano se ha enfrentado a diversas situaciones ya sean desconocidas o repetitivas que no le permiten tener comodidad al momento de desarrollar sus destrezas, por este motivo siempre está en constante cambio y en la búsqueda de soluciones a sus incomodidades, este tipo de situaciones le permiten desarrollar y crear habilidades nuevas que mejoren la situación a la que se enfrenta, por tal motivo, se puede mencionar que la creatividad es la capacidad que le permite a un individuo crear, imaginar, proyectar y desarrollar conocimientos. Para Esquivias (1997, como se citó en Esquivas, 2004), "La creatividad es un proceso mental complejo, el cual supone: actitudes, experiencias, combinatoria, originalidad y juego, para lograr una producción o aportación diferente a lo que ya existía” (p. 6).
Desde la mirada epistemológica, la creatividad según autores, López (1995, citado en Villamizar, 2012), afirma que:

Etimológicamente creatividad proviene de los términos latinos creativo y crear... Actualmente proviene del cristianismo, el cual concibió la creación del universo como producción divina a partir de la nada... (p. 213)

En concordancia con lo anterior, la creatividad permite la generación de nuevos saberes, los cuales facilitan la adaptación a cambios; para Yentzen (2003), menciona que:

Donde la creatividad surge, introduce una mirada diferente a lo existente, abriendo con ello la posibilidad de que surja lo nuevo... con el acto de creación, desde el más cotidiano hasta el acto mayor: es del Universo. (párr. 2)

En otras palabras, estas experiencias impulsan al individuo a mejorar las técnicas ya conocidas, buscando siempre que estas se vuelvan más fáciles y prácticas para su ejecución, esto permite que en el ambiente profesional se generen conocimientos y metodologías que provean el manejo de la información y creación de conocimientos, donde los informes y hechos ocurridos sean comprensibles y analizados, forjando la generación de nuevas estrategias para la optimización de los procesos que favorezcan el desarrollo de la profesión .

A partir de las concepciones teóricas anteriores a continuación se explicitan los conceptos que se relacionan con el objeto de estudio, y ayudan a contextualizar el desarrollo de la investigación; los cuales son fraude, ética profesional, principios generalmente aceptados del contador y quehacer contable.

En cuanto a el fraude, es una práctica que hoy en día es cada vez más común, se conoce como en- 
gaño y presentación de información errónea con el fin de conseguir algún beneficio, el cual por lo general es económico; en este sentido Galvis y Santos (2017), señalan que "Se puede definir el fraude como un engaño del que se obtiene un beneficio; esencialmente es el aporte de información inválida, incompleta o distorsionada que orienta la decisión de la víctima a favor del victimario" (párr. 7). El fraude en contabilidad, se refiere a la manipulación de estados financieros, con el fin de mostrar una imagen irreal de una entidad, para obtener beneficios económicos; como lo define Gil y Rodríguez (2010, como se citó en Ramírez y Reina, 2013), el fraude "es un fenómeno económico, social y organizacional, aplicado a la contabilidad; el fraude consiste en cualquier acto u omisión de un acto de naturaleza dolosa y por tanto de mala fe, o de negligencia grave" (p. 189).

Teniendo en cuenta lo expuesto por los autores, el profesional que cometa un hecho de materia dolosa, atenta directamente contra la ética profesional y los principios generalmente aceptados; cuando se habla de principios según el artículo 68 de la Ley 43 de 1990, señala que "se entiende por principios o normas de contabilidad generalmente aceptados en Colombia, el conjunto de conceptos básicos y de reglas que deben ser observados al registrar e informar contablemente sobre los asuntos y actividades de personas naturales o jurídica". De manera que se considera que un profesional de las ciencias contables, es una persona formada con valores éticos y competitivos, el cual tiene como obligación generar confianza y fe pública ante los usuarios, por lo tanto, esta persona se encuentra en la obligación de distinguir lo correcto de lo que no lo es y de encaminarse por lo real; Lisboa (2010, como lo cito Feil et al., 2017), esgrimen que "La ética profesional es fundamental en la conservación, seguridad, enriquecimiento, credibilidad, reconoci- miento, respeto y éxito en la profesión contable" (p. 259). De acuerdo con Lisboa, que el experto contable posee ética profesional, fiabilidad y credibilidad en la información que presenta ante sus usuarios, genera confianza ante estos; como ya se planteó anteriormente, el profesional debe ser oportuno con los informes que muestra, para Ferrel, et al. (2001, Como se citó en Feil et al., 2017), argumentan que "la ética profesional es un estándar de conducta basado en la moral, que se utiliza para distinguir lo aceptable de lo inaceptable" (p. 258).

\section{Marco Legal y Normativo}

En este apartado se presenta el marco normativo que sustenta el estudio, como base fundamental se sitúa la Constitución Política de Colombia de 1991, que es la norma de normas, esta rige en el territorio colombiano y que además posee todos los derechos y deberes de los habitantes de la $\mathrm{Na}$ ción. Esta Norma, da lugar a las leyes y decretos en las se fundamenta la investigación.

Bajo el objeto de esta pesquisa, de la Ley 43 de 1990, se toma como referencia el Capítulo IV Título I; por el cual se regulan los "principios que constituyen el fundamento esencial para el desarrollo de las normas sobre ética de la Contaduría Pública", donde el contador público como intermediario y agente de confianza debe contribuir de manera legal en la práctica de su profesión, aplicando los procedimientos adecuados conforme a la ley. De conformidad con lo antes expuesto, el estudio se ampara de la ley 1314 de 2009, por la cual se regulan los principios y normas de contabilidad e información financiera y de aseguramiento de información aceptados en Colombia, se señalan las autoridades competentes, el procedimiento para su expedición y se determinan las entidades responsables de vigilar su cumplimiento. 
Normalmente la disciplina contable se cobija en los lineamientos que suscita La Federación Internacional de Contadores (IFAC, por sus siglas en inglés, 2008), cuyo propósito es "promover la observancia de normas profesionales de alta calidad, impulsando la convergencia internacional de estas normas y tomando postura en el interés público allá donde la experiencia de la profesión sea más relevante" (p. 9).

Es de agregar dentro de las normas nacionales el Decreto 2649 de 1993, quien reglamenta la contabilidad en general y expide los principios o normas de contabilidad generalmente aceptados en Colombia, entendiéndose que toda persona que desea llevar contabilidad o hacer valer dicho documento debe regirse bajo este decreto.

Con relación a lo anterior, el artículo 581 del Decreto 624 de 1989, establece los efectos de la firma del contador, de acuerdo con los principios de contabilidad generalmente aceptados y con las normas vigentes sobre la materia, el cual los libros reflejan razonablemente la situación financiera de la empresa. Es denotar que el contador público al firmar, está aceptando y dando fe pública, que valida y proporciona, dando claridad que el acto respectivo se ajusta a las normas legales. De igual manera, para llevar a cabalidad el desarrollo de la investigación se toma como referente el módulo 2: conceptos y principios generales, de la Fundación de Normas Internacionales de Información Financiera (IFRS, por sus siglas en inglés, 2009), este documento fundamenta los principios de comparabilidad, transparencia y de calidad de tal manera que la contabilidad y los estados financieros contribuyan a los inversionistas, y usuarios, a tomar decisiones económicas.

El marco legal y normativo mencionado con anterioridad, contribuye a orientar al profesional y a la sociedad interesada, en el panorama con el que se encuentra regida y amparada la profesión contable.

\section{Marco Contextual}

Para establecer el marco contextual se indagaron y revisaron alrededor de 28 investigaciones recopiladas de revistas indexadas en bases de datos, en donde se pudo analizar la concepción de la palabra creatividad y la percepción que se tiene en la actualidad sobre la contabilidad creativa, además como se ha enrarecido el concepto de creatividad, llevándolo a la homologación con el termino de fraude.

El contador público, hoy día y teniendo en cuenta las normas internacionales de la profesión, debe ser un individuo fundamentado en principios que acrediten su ética profesional, es por esto que la contabilidad creativa debe ir encaminada hacia la generación de conocimiento e innovación y no al aprovechamiento de esta para generar hechos de dolo; la contabilidad creativa en este caso suele ser flexible, de acuerdo con Casabianca (2005), quien sostiene que "es difícil delimitar dónde acaba la ética y empieza la creatividad y dónde termina ésta y empieza el fraude, y en definitiva, ¿cuál es el límite entre la contabilidad creativa y el fraude?" (p. 57), como lo hace notar Casabianca, resulta complejo determinar cuál es este punto, al percibir que la creatividad le permite llegar a un individuo hasta donde él esté dispuesto, el verdadero problema es la interpretación y manejo que le dé realmente; al pasar esa pequeña línea de lo correcto a lo incorrecto, es cuando se le está dando otro significado, el cual aparentemente es el que se está viendo hasta la fecha de hoy.

Tal como lo expone Guevara y Consenza (2004), manifiestan que "en la actualidad es difícil distinguir, en forma clara, la frontera que separa la llamada CC del fraude o delito contable" (p. 7). Como anteriormente señala el autor, hoy en día es muy difícil explicar si la contabilidad creativa son conocimientos aplicados para mejorar el sistema 
contable o un método que permite al profesional jugar con las normas de manera inadecuada faltando a los principios de contabilidad generalmente aceptados, de manera que no es vista únicamente desde las habilidades y el conocimiento que posee el ser humano para mejorar e innovar.

\section{Metodología}

Para el desarrollo del objeto de estudio se plantea una investigación de enfoque cualitativo, cuya orientación según Hernández et al. (2004, Como se citó en Cuenya y Ruetti, 2010):

Se fundamentan más en un proceso de exploración y descripción, y luego se generan perspectivas teóricas. La investigación cualitativa se apoya sobre la idea de la unidad de la realidad, de ahí que sea holística, y en la fidelidad de la perspectiva de los actores involucrados en esa realidad. (p. 273)

Este enfoque adquiere importancia, porque se aproxima a la realidad de lo sujetos, que para el caso de esta investigación son los profesionales contables.

La investigación se desarrolla con el método descriptivo; porque la información recabada pasa por un proceso de identificación, análisis y construcción que dé respuesta a la pregunta y al desarrollo de los objetivos. De acuerdo a lo antes expuesto, para Jorba et al. (2000, Como se citó en Murillo y Martínez, 2014):

Esta habilidad consiste en producir proposiciones o enunciados que enumeren propiedades del objeto o fenómeno, ayuda a procesar información con las propiedades, características y cualidades de los fenómenos, objetos y hechos sociales. Su objetivo es informar sobre cualidades que identifiquen lo esencial de una realidad o fenómeno. (p. 107)
La unidad de análisis con la que se desarrolla el estudio, son los profesionales contables, contadores públicos en ejercicio y en formación de la ciudad de Villavicencio, capital, centro económico y comercial del departamento de Meta, en un contexto empresarial que cuenta, según el directorio de empresas de Colombia del portal de $\mathrm{E}$ Informa-información de empresas. (s.f.), quien menciona que en esta zona geográfica hay aproximadamente 9.698 empresas actualmente. Además, se tienen presentes los sujetos activos de la profesión que se desempeñan en esta ciudad y las 6 Universidades que ofrecen el programa de Contaduría Pública; que de manera consensuada acepte participar en la investigación y además estará conformada por producción intelectual fruto de investigaciones acerca del objeto de estudio.

Cabe mencionar, que cuando se hace referencia a unidad de análisis según Melian y Picón (2014), se habla de una organización categórica con la cual se puede dar respuesta a la pregunta formulada para dar solución al objeto de estudio; este se compone de un material práctico relacionado con el problema y además de una estructura soportada en teorías con las cuales se puede deducir con más seguridad la respuesta de un proyecto.

Los instrumentos que se emplean para el desarrollo y la obtención de la información, son entrevistas como herramienta para la investigación cualitativa, la cual de acuerdo con López y Deslauriers (2011), es un recurso que se ha utilizado a lo largo de los años, por el cual se lleva a cabo la comunicación entre dos partes, de la cual una de ellas adquiere información o datos de la otra. De este modo, se aplicará esta herramienta a la unidad de análisis la cual estará integrada por los profesionales contables ya sean en ejercicio o en formación, que se encuentren en la ciudad de Villavicencio, los cuales expresen su percepción e interpretación de contabilidad creativa. 
De igual modo, se realiza análisis de documentos, de la información obtenida sobre investigaciones desarrolladas que aborden el tema de contabilidad creativa; de acuerdo con Gómez et al. (2017), "la revisión documental, como herramienta ayuda en la construcción del conocimiento, amplia los constructos hipotéticos de los estudiantes y como enriquece su vocabulario para interpretar su realidad desde su disciplina..." (p.53).

\section{Reflexión}

La contabilidad es una disciplina muy antigua, que a través del tiempo ha ido evolucionando a medida que la sociedad lo va requiriendo, implementado sistemas que permitan llevar con mejor exactitud la información financiera. Sin embargo, es de mencionar que la contabilidad es una profesión que demanda diversas áreas contables, donde una de ellas es la contabilidad creativa, quien actualmente ha empezado a formar parte del lenguaje contable, de una manera inadecuada, debido a que su concepto fue homogenizado con el de fraude; donde el profesional realiza actos inapropiados, para el beneficio de sí mismo o de terceros; deplorando la credibilidad y confianza que depositan los usuarios que intervienen en la revisión de la información financiera.

Las investigaciones realizadas, abordan perspectivas de distintos autores sobre el concepto de contabilidad creativa, donde se encontraron definiciones muy similares, pero al realizar un bosquejo sobre el significado de creatividad esta nos conduce a obtener otra apreciación, en el cual se podría decir que esta área contable ha sido mal interpretada desde hace mucho tiempo.

Esta investigación tiene como intención poner de manifiesto el concepto real y el que la sociedad tiene sobre la contabilidad creativa, en donde actualmente sigue siendo comprendida de forma errónea, causando perjuicio a la credibilidad del profesional, según autores como Amat y Oliveras (2004) "si los reguladores de la normativa y la profesión contable trabajan juntos pueden encontrar vías para combatir eficazmente la contabilidad creativa" (p.16). Con respecto a lo antes mencionado, más allá de ser un control interno en la normatividad, entra a jugar un papel clave que es la ética profesional de quien realiza la práctica contable.

En definitiva, el experto contable carga sobre sus hombros una responsabilidad y una obligación inmensa debido a que este tiene un compromiso con su integridad moral, con su entorno profesional, con la organización a la que presta sus servicios y con la sociedad. En donde cualquiera de sus acciones u omisiones desempeñadas, pueden llegar a ser consideradas como un acto de mala fe o negligencia, por todo esto el profesional siempre estará expuesto a diferentes situaciones, pero es allí donde él examina de acuerdo a sus conocimientos, experiencias y competencias adquiridas y decide discernir en lo que es favorable o no para su crecimiento laboral, personal, familiar y ante la sociedad, porque es de gran importancia mencionar las sanciones en las que puede incurrir hasta la inhabilidad para ejercer la profesión y darle punto final a muchos años de dedicación y esfuerzo. 


\section{Referencias}

Abbate, E., Mileti, M. y Vázquez, C. (2000). La necesidad de investigar en contabilidad. Invenio, 3(4-5), 115 - 124. https:/ / www. redalyc.org/pdf/877/87730508.pdf

Amat, O. y Oliveras, E. (2004). Propuestas para combatirla contabilidad creativa. Universia Business Review. (1) 10-17. https://www. redalyc.org/pdf/433/43300101.pdf

Arbeláez, D. F., Correa, L. y Silva, J. (2013). Un acercamiento a los desarrollos investigativos en la auditoría forense. Tendencias, 14(2), 216-230. https: / dialnet.unirioja. es/servlet/articulo?código $=4722794$

Bravo, S. (2011). La contabilidad creativa. AECA: Revista de la asociación española de contabilidad y administración de empresas, (94), 1-63. http:/ / www.aeca1.org/revistaeca/revista94/94.pdf

Casabianca, M L. (2005). Contabilidad creativa. Revista FCE, 1(3), 57-63. https://dialnet.unirioja.es/servlet/ articulo?codigo $=5523081$

Constitución Política de Colombia. (1991, 4 de julio). Asamblea Nacional Constituyente de Colombia. Diario oficial No 51445. http://wsp.presidencia.gov.co/ Normativa/Documents/ConstitucionPolitica-Colombia.pdf

Cuenya, L., Ruetti, E. (2010). Controversias epistemológicas y metodológicas entre el paradigma cualitativo y cuantitativo en psicología. Revista colombiana de psicología, 19(2), 271-277. https:/ / dialnet.unirioja. es/servlet/articulo?codigo $=3641525$
Decreto 624 de 1989. (1989, 30 de marzo). Presidente de la Republica de Colombia. Artículo 581. Diario Oficial No 38756. http://www.suinjuriscol.gov.co/viewDocument. asp?ruta $=$ Decretos $/ 1132325$

Decreto 2649 de 1993. (1993,29 de diciembre). Presidente de la Republica de Colombia. Diario Oficial No 41156. http:/ /www.suin-juriscol.gov.co/viewDocument.asp?id=1476299

E Informa-información de empresas. (s.f.). Directorio de empresas de Colombia. https: / / directorio-empresas.einforma. co/localidad/villavicencio/

Esquivas, M T. (2004). Creatividad: Definiciones, antecedentes y aportaciones. Revista digital universitaria RDU, 5(1), 1-17. https://www.revista.unam.mx/vol.5/ num $1 /$ art4/ene_art4.pdf

Feil, A., Diehl, L. y Schuck, R. (2017). Professional ethics and accounting students: analysis of the intervening variables. Cuadernos EBAPE. $B R$, 15(2), 256- 273. https://www. scielo.br/scielo.php?pid=S167939512017000200256 \&script $=$ sci_ arttext\&tlng=en

Flores, I., Flores, R. y Aguiar, J. (2013). La teoría del conocimiento y epistemología de administración. Xikua, 1 (2). https://www.uaeh.edu.mx/scige/boletin/tlahuelilpan/n1/e4.html 


\section{Referencias}

Fundación IFRS (2009). NIIF 2 para Pymes: Conceptos y principios generales. https:// www.icicat.co/normatividad/finanzas/normas-internacionales/niif-parapymes/seccion-2-conceptos-y-principios-generales

Galvis, I. y Santos, J. (2017). Geometría del fraude. Cuadernos de contabilidad, 18(45). https:// dialnet.unirioja.es/servlet/ articulo? codigo $=6409424$

García, R. (2006). Epistemología y teoría del conocimiento. Salud colectiva, 2(2), 113122. https://www.scielosp.org/article/ scol/2006.v2n2/109-122/es/

Gerencie.com (2012,09 de febrero). La ética del Contador Público. https://www.gerencie.com/la-etica-del-contador-publico.html\#: :text $=\mathrm{La} \% 20$ norma $\% 20$ $\mathrm{m} \%$ C 3\% A 1 s \% 20 vi ola d a $\% 20$ de,para $\% 201$ los $\% 20$ profesionales $\% 20$ $\mathrm{de} \% 201 \mathrm{a}$

Gómez, D., Carranza, Y. y Ramos, C. (2017). Revisión documental, una herramienta para el mejoramiento de las competencias de lectura y escritura en estudiantes universitarios. Chakinan, (1), 46 - 56. http://scielo.senescyt. gob.ec/scielo.php? script=sci_arttext\&pid=S2550-67222017000300046

Gómez, M. (2008). Dinámica de la concepción y la enseñanza de la teoría contable en Colombia (1970-2000): una exploración institucional. Facultad de Ciencias Económicas, Universidad Nacional de Colombia. http:/ / www.bdigital.unal.edu.co/762/2/ Doc_Gomez_EACP3.pdf

Guevara, I R. y Consenza, J P. (2004). Los auditores independientes y la contabilidad creativa: estudio empírico comparativo. Compendium, (12), 5-24. https://dialnet.unirioja.es/servlet/articulo? codi$\mathrm{go}=2955150$

Federación Internacional de Contadores, IFAC (2009). Código de ética para profesionales de la contabilidad. https://www.ifac.org/system/files/ publications/files/codigo-de-etica-para-profesionales-de-la-contabilidad.pdf

Ley 43 de 1990. (1990,13 de diciembre). El congreso de la Republica. Diario oficial No 39602. http:/ /www.suin-juriscol.gov.co/viewDocument.asp? ruta=Leyes $/ 1598256$

Ley 1314 de 2009. (2009,13 de julio). Congreso de la Republica. Diario Oficial No 47409. http://www.suin-juriscol.gov. co/viewDocument.asp? ruta=Leyes / 1677255\#: :text=LEY\%201314\%20 DE $\% 202009 \&$ text $=($ julio\%2013) ,por $\% 201 \mathrm{a} \% 20$ cual $\% 20$ se $\% 20$ regulan $\% 201$ los $\% 20$ principios $\% 20 y \% 20$ normas $\% 20$ de,responsables $\% 20$ de $\% 20$ vigilar $\% 20$ su $\% 20$ cumplimiento

López, R. y Deslauriers, J. (2011). La entrevista cualitativa como técnica para la investigación en trabajo social. Margen: revista de trabajo social y ciencias sociales, (61), 1- 9. https://dialnet.unirioja.es/ servlet/articulo? codigo $=3756178$ 


\section{Referencias}

Mejía, E., Montilla, O. y Montes, C. (2010). Realismo científico y contabilidad: Una evaluación a la disciplina contable. Entramado, 6 (1), 56-75. https://dialnet.unirioja.es/servlet/ articulo? codigo $=3823467$

Melian, Y. y Picón, D. (2014). La unidad de análisis en la problemática enseñanza-aprendizaje. Informe científico técnico UNPA, 6(3), 101 - 117. https://dialnet.unirioja.es/servlet/ articulo? codigo $=5123550$

Murillo, J A. y Martínez, C A. (2014). Habilidades de pensamiento social: describir, explicar, interpretar y argumentar en el aula. Itinerario educativo, (64), 108-125. https://dialnet.unirioja.es/servlet/ articulo? codigo $=6280214$

Ospina, C A. (2004). Disciplina, saber y existencia. Revista latinoamericana de ciencias sociales, niñez y juventud, 2(2), 122. http://www.scielo.org.co/scielo. php?script $=$ sci_arttext\&pid=S1692715X2004000200003

Pérez, V G. y Pinto, G R. (2016). La investigación contable como herramienta didáctica en la docencia de la contabilidad. Ciencias administrativas, (7). https://revistas.unlp.edu.ar/CADM/ article/view/2037
Ramírez, A. (2009). La teoría del conocimiento en investigación científica: una visión actual. Anales de la facultad de medicina, 70 (3), 217- 224. http://www.scielo.org. pe/scielo.php?script=sci_arttext\&pid $=$ S1025-55832009000300011

Ramírez, M. y Reina, J. (2013) Metodología y desarrollo de la auditoria forense en la detección del fraude contable en Colombia. Cuadernos de administración, 29(50), 186-195. http://www.scielo.org.co/scielo. php?script $=$ sci_arttext\&pid $=$ S012046452013000200008\&lang=es

Santos, M. (2016). La contabilidad creativa, el directivo y la ética organizacional. Retos de la dirección, 10(2), 46-67. http://scielo.sld.cu/scielo.php?script=sci_arttext \&pid=S2306-91552016000200004

Villamizar, G. (2012). La creatividad desde la perspectiva de estudiantes universitarios. Reice, 10 (2), 212-237. https://www.redalyc.org/pdf/551/55124596015.pdf

Villareal, J L. (2009). Bases conceptuales para la construcción de la teoría contable. Criterio libre, 7(11), 167- 190. https://dialnet.unirioja.es/servlet/ articulo? codigo $=3227606$

Yentzen, E. (2003). Teoría general de la creatividad. Polis: Revista Latinoamericana, 2(6). https://www.redalyc.org/ pdf/305/30500612.pdf 